\title{
Cesarean scar pregnancy: tertiary-centre experience
}

\begin{abstract}
Background: Past history of previous Cesarean delivery [CD] carries the risk for gestational sac implantation of any subsequent pregnancy over the cesarean scar developing what is defined as a cesarean scar pregnancy [CSP]. The aim of this article was to evaluate possible interventions used for the diagnosis and treatment of CSP.

Materials and methods: This cohort analysis of case series included all patients admitted to Obstetrics and Gynecology department, King Faisal Military Hospital over a period of 5 years [2014 through 2019 and evaluated in 2019] with the diagnosis of suspected CSP. Demographic characteristics, clinical and outcome data were obtained from the original electronic hospital charts, operation notes, anesthesia notes, discharge summaries, nursing notes and outpatient medical records.

Results: During the study period 12 patients were diagnosed by US as having CSP, the mean age was 36 years, the mean of parity was 4.7. Most of the patients had previous two CD [33\%]. The mean time interval between the previous CD and the current CSP was 16.4 months and the estimated gestational age was 46 days. The mean diameter of the mass in the sac of CSP was $9.7 \mathrm{~mm}$, and the mean of serum $\beta$-hCG levels was $53765.8 \mathrm{mIU} / \mathrm{mL}$. All patients were successfully treated by methotrexate and the mean resolution time was 52 days.
\end{abstract}

Conclusion: Methotrexate [MTX] was effective for treatment of CSP but larger multicenter studies with large number of patients are necessary to confirm our results.
Volume 8 Issue 3 - 2019

\author{
Ahmed Altraigey,' Ahmed Badawie, ${ }^{2,3}$ Sara \\ Taha Mostafa' \\ 'Department of Obstetrics and Gynaecology, Benha University, \\ Benha, Egypt \\ ${ }^{2}$ Department of Obstetrics and Gynaecology, October 6 \\ University, Giza, Egypt \\ ${ }^{3}$ Department of Obstetrics and Gynecology,Armed Forces \\ Hospital Southern Region, Saudia Arabia
}

Correspondence: Ahmed Altraigey MD, Department of Obstetrics and Gynaecology, Benha University, 43 benha zagzig street, Mansheyet Elnoor, Benha, Egypt, Tel +966544854232, Email ahmed.altraigey@yahoo.com

Received: May 02, 2019 | Published: May 29, 2019

\section{Introduction}

The Cesarean delivery $[\mathrm{CD}]$ rate increased markedly in the past two decades. Its rate was doubled between 2000 and 2015 to reach almost $21 \%$ of all live births. This increase was noticed in 169 countries that reported 29.7 million deliveries by CD annually. ${ }^{1}$ This raise could be explained by the rise of primary [first] CD [from 12.6$20.6 \%$ ] and a decline in vaginal births after CD [28-9.2\%], so that the rate of repeat $\mathrm{CD}$ is now about $91 \% .^{2}$ The maternal morbidity prevalence is higher after $\mathrm{CD}$ than after normal vaginal birth. $\mathrm{CD}$ is associated with a higher incidence of ectopic pregnancy, abnormal placentation [placenta previalaccreta] and uterine rupture. Moreover, these risks increased in a dose-response manner. ${ }^{3}$ History of a past $\mathrm{CD}$ increased the risk of gestational sac implantation of the next pregnancy over the cesarean scar, creating the clinical condition defined as cesarean scar pregnancy [CSP] and as explained the magnitude of this risk raised with more repeated CD. ${ }^{4}$ Two types of CSP were recognized according to the extent of gestational sac invasion; Type 1: where superficial implantation on a scar progressing subsequently towards the cervico-isthmic space and/ or the uterine cavity, whereas Type 2: direct deep implantation into the myometrium \pm reaching up to inner surface of uterine visceral serosa. ${ }^{5}$ Since 2000, CSP incidence showed a significant increase, up to $6.1 \%$ of all ectopic pregnancies in women with past history of $\mathrm{CD}$, which might be attributed to both increased number of CD and the improved diagnostic accuracy tools recognizing ectopic pregnancy ${ }^{6} \mathrm{CSP}$ carried the risk of major bleeding, fatal hemorrhage, and spontaneous uterine rupture up to hysterectomy to save the women's lives. ${ }^{7}$ Although its clinical presentation vary between light painless vaginal bleeding and moderate abdominal pain, the accurate diagnosis remained difficult as the false negative results of multiple tests could lead to a life-threatening scenario. ${ }^{8}$ The ideal pathway of CSP management is widely controversial. However, it is universally agreed that surgery is the unavoidable 1st line of management of women presenting with uterine rupture or severe bleeding. On the other hand, the management of hemodynamically stable diagnosed with CSP might represent a challenge. There were almost 31 primary approaches published to treat CSPs but mostly sporadic and individual cases and their results seemed to be insufficient to conclude clearly which one was the most effective management protocol that leaded to the least adverse events. Thus, there is increased needs to develop a set of practice guidelines for health care professionals considering optimum management of CSP. The aim of this work was to describe the experience of a tertiary care hospital with the diagnosis and treatment of CSP and to explore patients' complications related to this rare type of ectopic pregnancy.

\section{Patients and methods}

This Cohort study was conducted in King Faisal Military Hospital, Khamis Mushayt, Southern Region, Saudi Arabia in the period from June 2015 to January 2019 after obtaining the approval of the local institutional ethics and research committee [Armed Forces Hospital Southern Region ethics and research committee]. All patients admitted to Obstetrics and Gynecology Department, King Faisal military hospital over a period of 5 years [2015-2019] with the diagnosis of suspected CSP were included. Hemodynamic instability that required immediate surgical intervention was the only exclusion criteria. All patients were consented before starting data collection and after full counselling about the hysterectomy risk. All patients accepted the conservative treatment including methotrexate injection, D\&C, or laparotomy and wedge resection of the scar ectopic pregnancy because they all asked for fertility preservation. When CSP was suspected by a thorough history taking, including obstetric, reproductive, surgical history as well as physical examination, the diagnosis was confirmed by the following US findings: (Figure 1) 

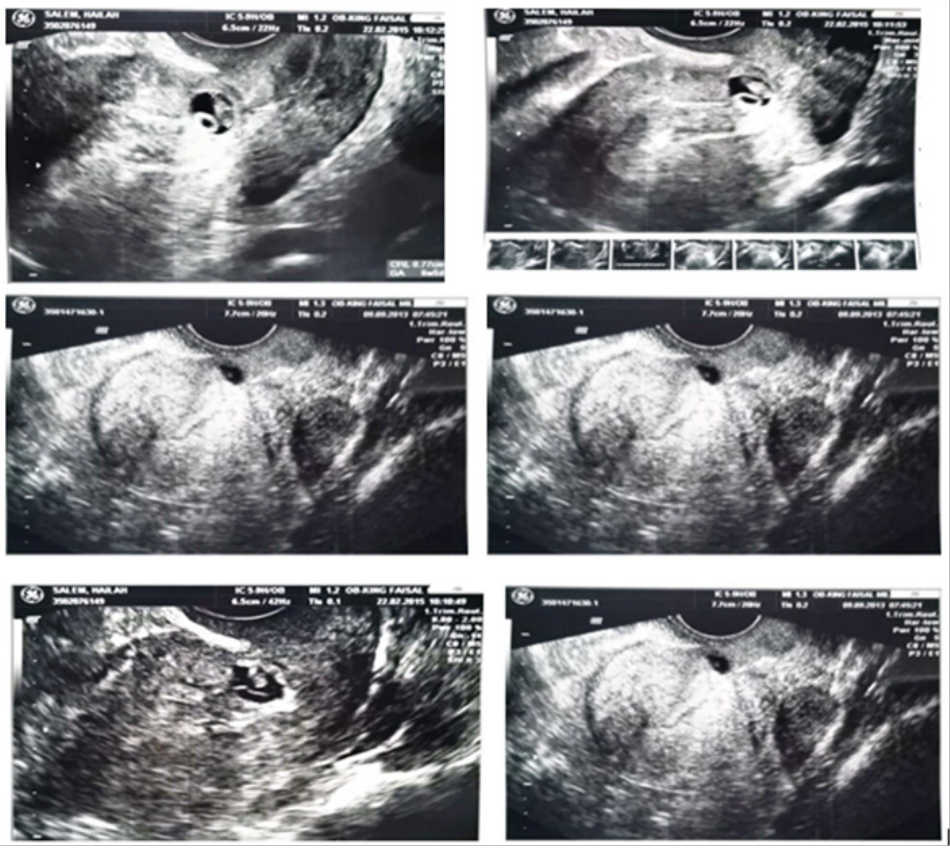

Figure I Ultrasound findings conclusive of CSP; mainly the gestational sac is embedded inside myometrium and empty uterine cavity.

a) Empty uterine cavity

b) The placenta or gestational sac impedance in the cesarean scar area

c) A triangular gestational sac that fills the scar niche in early gestations $[\leq 8$ weeks] that change to become rounded or even oval at $>8$ weeks

d) Thin $[<4 \mathrm{~mm}]$ or absent myometrial layer between the gestational sac and the bladder

e) Closed and empty endocervical canal

f) Embryonic/fetal pole and/or yolk sac with or without heart activity

g) Prominent [rich] vascular pattern at or in the area of a CD scar

Data were abstracted from the original hospital charts, operation notes, anesthesia notes, discharge summaries, nursing notes and outpatient medical records. From each patient's file, the following data were searched for age, parity, presenting symptoms, number of previous $\mathrm{CD}$, duration from last cesarean section, gestational age at diagnosis, fetal cardiac activity, initial diagnosis [e.g. early pregnancy, cervical pregnancy, incomplete miscarriage], B-hCG concentration [at admission and then at different times of follow-up], management plan, complications [whether or not related to the management] and time interval needed for $\beta$-hCG to return back to normal. Analysis of the data was done by IBM computer using SPSS [statistical product and service solution version 21]. Quantitative variables were described as mean with range while qualitative variables will be described as numbers and percentages.

\section{Results}

During the study period 12 patients were diagnosed by US as having CSP, the range of the age was between 32 and 45 years old. The lowest parity was 1 and the highest was 10 . The mean $[ \pm \mathrm{SD}]$ of body mass index [BMI] was $30.8 \pm 4.4 \mathrm{~kg} / \mathrm{m} 2$. The interval between recent pregnancy and the last delivery ranged from 10 months to 24 months. It needed between 33 days and 77 days gestational age to diagnose CSP. All women got pregnant spontaneously and $33 \%$ of them had previous two $\mathrm{CD}$ as the highest frequency as shown in Table 1. 58.3\% women experienced abdominal pain, while 33\% were identified asymptomatically as an incidental finding. The mean $[ \pm \mathrm{SD}]$ of serum pretreatment $\beta$-hCG levels and hemoglobin were [20432.5 \pm 21082.7 $\mathrm{mIU} / \mathrm{mL}, 11.9 \pm 1.5 \mathrm{gm} / \mathrm{dl}$ respectively]. All women were diagnosed solely using ultrasound [US] without any need for extra imaging tools. No women were diagnosed with intra-peritoneal fluid. Five women $[42 \%]$ showed signs of fetal cardiac activity Table 2 . The whole cohort received methotrexate therapy as the 1 st line treatment which was successful initially in seven women [58.3\%], while the rest needed extra course of same medication. It needed around $300 \mathrm{mg}$ and 58 days of methotrexate to achieve effective decline of $\beta$-hCG Table 3 . Only one patient on the 5th day following Methotrexate [MTX] injection suffered from excessive vaginal bleeding and surgical interference was mandatory to save the patients life, so emergency suction curettage under US guidance was performed. All patients showed no drug side effects.

Table I Characteristics of women under the study

\begin{tabular}{lll}
\hline Variable & Mean \pm SD \\
\hline Women age [years] & $36.1 \pm 4.1$ \\
Parity & $4.7 \pm 2.6$ \\
BMI [kg/m2] & $30.8 \pm 4.4$ \\
Interval from last pregnancy [months] & $16.4 \pm 5.7$ \\
Gestational age at diagnosis [days] & $46.0 \pm 12.3$ \\
Variable & & Frequency [\%] \\
& Spontaneous & $12[100 \%]$ \\
Mode of conception & Assisted & $0[0]$ \\
Number of previous CD & One & $3[25 \%]$ \\
& Two & $4[33 \%]$ \\
& Three & $3[25 \%]$ \\
& Four & $2[17 \%]$ \\
\hline
\end{tabular}

SD, Standard Deviation 
Table 2 Clinical characteristics of women under the study

\begin{tabular}{lll}
\hline Variable & Mean \pm SD \\
\hline$\beta$-hCG at admission & $20432.5 \pm 21082.7$ \\
Hemoglobin at admission [gm/dl] & $11.9 \pm 1.5$ \\
Size of the mass [mm] & $9.7 \pm 4.2$ \\
Variable & Frequency [\%] \\
& Abdominal pain & $7[58.3 \%]$ \\
Clinical presentation & $\begin{array}{l}\text { Vaginal } \\
\text { bleeding }\end{array}$ & $1[8.3 \%]$ \\
Presence of fetal cardiac & Asymptomatic & $4[33.3 \%]$ \\
pulsations & Present & $5[41.7 \%]$ \\
& Absent & $7[58.3 \%]$ \\
Presence of intra-peritoneal & Present & $0[0]$ \\
hemorrhage & Absent & $12[100 \%]$ \\
\end{tabular}

SD, Standard Deviation

Table 3 Outcome measures of women under the study

\begin{tabular}{|c|c|c|}
\hline \multicolumn{2}{|l|}{ Variable } & Mean士SD \\
\hline \multicolumn{2}{|c|}{ Total dose of MTX given [mg] } & $350 \pm 172.9$ \\
\hline \multicolumn{2}{|c|}{ Time to normalization of $\beta$-hCG [days] } & $52.5 \pm 12.9$ \\
\hline \multicolumn{2}{|l|}{ Variable } & Frequency [\%] \\
\hline \multirow[t]{2}{*}{$\begin{array}{l}\text { Number of methotrexate } \\
\text { courses }\end{array}$} & a) One course & $7[58.3 \%]$ \\
\hline & b) 2 course & 5 [41.7\%] \\
\hline \multirow[t]{2}{*}{ Complications } & a) Present & I [8.3\%] \\
\hline & b) Absent & II [91.7\%] \\
\hline
\end{tabular}

SD, Standard Deviation

\section{Discussion}

Cesarean scar pregnancy is considered as a rare form of ectopic pregnancy, and its incidence in our hospital may be increased due to the rise of the rate of Cesarean sections performed. The incidence of Cesarean scar pregnancy was 1: 2216 [12 cases/26,596 women at the study period] in this study and its rate was $6.1 \%$ [12 scar pregnancies/198 ectopic pregnancies] with a history of at least one previous Cesarean section. The incidence of Cesarean scar pregnancy was $0.15 \%$ [ 12 cases $/ 7980 \mathrm{CD}$ ] in women with previous $\mathrm{CD}$. There are two explanation of this rise in our hospital. Firstly, being a tertiary referral center of a local district where many women suffering abnormal or ectopic pregnancies were referred for management. Second, the higher frequencies the transvaginal US provided allowed early diagnosis of abnormal pregnancies. Increased recognition and the number of $\mathrm{CD}$ are presumably responsible for that rise in numbers of CSP. The natural history of CSP condition is unknown, but spontaneous scar rupture and hemorrhage, even early as in the first trimester, if the pregnancy is permitted to resume, with potential dangerous maternal adverse events. Myometrial and endometrial scarring or disruption might lead to implantation abnormality. Also, trophoblast invasion or adherence increased due to the scanty decidualization of the lower uterine segment. However, pregnancy implantation within the scar of a previous CD differed from pregnancy with placenta accreta. Implantation site of CSP is completely surrounded by myometrium and the scar fibrous tissue and totally separated from empty endometrial cavity. The mechanism that mostly could explain implantation within the fibrous scar is myometrial invasion through a microscopic tract. This tract is thought to be due to the surgical trauma of previous uterine procedure like curettage, manual removal of the placenta, $\mathrm{CD}$, myomectomy and even hysteroscopy. ${ }^{9}$ The time elapsed between trauma and the following pregnancy had an impact on implantation as some reported cases were detected within months of a prior $\mathrm{CD}$ which suggested that if uterine scar was incompletely healed, it contributed to scar implantation. ${ }^{10}$ Veridiano ${ }^{11}$ described a case complicated by uterine perforation during curettage at 14 weeks of gestation due to placenta percreta in a uterine scar. An emergency hysterectomy was performed following profuse bleeding. Herman ${ }^{12}$ described the first case detected using US that revealed an early gestational sac implanted inside a uterine scar. Their case was allowed to follow up her pregnancy that ended by emergency $\mathrm{CD}$ and hysterectomy due to severe bleeding that preceded intravascular coagulopathy. Early diagnosis is critical for offering multiple treatment options so that young women who desire to preserve fertility can be managed conservatively. Although diagnosis of ectopic pregnancy by US was reported more than four decades ago, the use of US in detecting a CSP was not reported until $1990 .{ }^{13}$ Strict criteria in US imaging must be used to differentiate CSP from spontaneous abortion in progress, cervico-isthmican empty uterine cavity, an empty cervical canal, gestational sac located in the anterior part of the uterine isthmus, and an absence of healthy myometrium between the bladder and the sac. ${ }^{14}$ Some authors suggested an additional criterion of prominent peri-trophoblastic flow in the diagnosis of CSP. ${ }^{15}$ However, misdiagnoses still occur. Lai ${ }^{16}$ reported difficulty in making a differential diagnosis between an ectopic pregnancy implanted in the oviduct or in the myometrium of a previous CSP, even as early as the 7th week of gestation. They suggested operative laparoscopy as a diagnostic tool. On the other hand, Godin et al reported the use of MRI to confirm a diagnosis of CSP. ${ }^{17} \mathrm{MRI}$ or three dimensional-colors Doppler imaging can enhance the diagnostic accuracy by evaluating the flow, resistance and pulsatility indices in the peri-trophoblastic vasculature; however, images may still resemble placenta accreta later in pregnancy. ${ }^{6}$ Because of the rarity of the condition, the majority of CSPs are case reports or small case series reported in the literature, with no consensus on the preferred mode of treatment. Generally, termination of pregnancy in the first trimester is strongly recommended to avoid lifethreatening complications. ${ }^{18}$ Treatment objectives should be to perform feticide prior to rupture, to remove the gestation sac and to retain the patient's future fertility. MTX has been used extensively as first-line treatment in cases of tubal and cervical pregnancy if gestational age is less than 9 weeks, fetal pole size does not exceed 10 $\mathrm{mm}$, no embryonic cardiac activity is seen, and serum $\beta$-hCG levels are less than 10,000 IU/L. ${ }^{19}$ Experience regarding the efficacy and dosage is limited in CSP. A single dose of systemic MTX has been used to treat CSP. ${ }^{20,21}$ However, others have claimed that systemic multi-doses of MTX are necessary for successful management. ${ }^{22,23}$ Such treatment was initially employed for the management of CSP as an adjunct to hysterotomy or other procedures. Later, however, it was used as a primary therapy, for cases diagnosed early. Slow drug absorption into the CSP after systemic methotrexate is expected because the pregnancy is surrounded by fibrous scar rather than a 
normally vascularized myometrium, potentially limiting systemic access. In our study, all patients responded well to systemic MTX. Rotas et al., ${ }^{8}$ reported 16 cases that were treated only by systemic intramuscular methotrexate; in 5 [36\%], all with baseline $\beta$-hCG level less than $5,000 \mathrm{mIU} / \mathrm{mL}$, it led to a complete and uncomplicated resolution within a few months. Another 5 women received multiple doses of intramuscular MTX alternating with leucovorin. Treatment was successful in 3 of the women; in the remaining 2 women, it was complicated by hemorrhage that was managed by laparotomy and wedge resection in one and hysterectomy in the other. The mean resolution time for CSP in our study was 52 days [range 27-67 days]. $\mathrm{Wu}^{24}$ found the decrease of serum $\beta$-hCG levels in patients receiving intramuscular MTX injection was slow, and only four [16\%] patients had a $50 \%$ drop in their $\beta$-hCG levels after the first dose of MTX. The study by Yang $^{25}$ also found the time for $\beta$-hCG level to decline to normal was long in CSP patients receiving intramuscular MTX injection only. Since the decline of $\beta$-hCG level may very slow, and uncontrolled vaginal bleeding could take place or continue at any time, and it was hard to predict when the CSP mass completely resolved if treated with MTX only. Only one treatment course of MTX was needed in 7 of our patients [58\%], while two courses were needed in the remaining 5 [42\%]. Using serum $\beta$-hCG level as an indicator for the need for further treatment, $\mathrm{Chen}^{26}$ found a $68.3 \%$ decline of $\beta$-hCG level in one case, but only a decline of $49.6 \%$ in the other case, 1 week after treatment, levels of $\beta$-hCG were high [33,082 $\mathrm{mIU} / \mathrm{mL}$ ] after treatment in the later case, prompting two additional courses of systemic MTX, while the first case received only one course of systemic MTX therapy. These results suggested that the number of treatment courses required might be related to gestational age at diagnosis and to the presence of a fetal heartbeat. Before MTX treatment, the mean of serum $\beta$-hCG levels of our patients was $13765.8 \mathrm{mIU} / \mathrm{mL}$. Unlike the experience with single-dose MTX treatment in tubal pregnancies that indicate a higher failure rate for patients with an initial $\beta$-hCG level greater than $10,000 \mathrm{mIU} / \mathrm{mL}$. Fertility outcome after treatment of ectopic pregnancy is one of the key factors when choosing treatment strategy. We did not asses the overall success rate or the outcome of subsequent pregnancies after treatment for CSP. Several authors have reported uneventful subsequent pregnancy course after surgical repair, ${ }^{27}$ but the integrity of the uterus after medical treatment is still unclear. Finally, although the medical treatment of CSP of our 12 patients was successful, the evaluation of treatment results based on the outcome of only 12 cases is not sufficient to draw a final conclusion regarding the efficacy of such treatment modality, especially also because there was no direct comparison with other available treatment options. So in view of the increasing rate of CS, health care providers should be aware of the possibility of this ectopic pregnancy type in subsequent pregnancies.

\section{Acknowledgments}

None.

\section{Conflicts of interest}

The author declares there are no conflicts of interest.

\section{References}

1. Boerma T, Ronsmans C, Melesse DY, et al. Global epidemiology of use of and disparities in caesarean Sections. Lancet. 2018;392(10155):13411348.
2. Betrán AP, Temmerman M, Kingdon C, et al. Interventions to reduce unnecessary caesarean sections in healthy women and babies. Lancet. 2018;392(10155):1358-1368.

3. Sandall J, Tribe RM, Avery L, et al. Short-term and long-term effects of caesarean section on the health of women and children. Lancet. 2018; 392(10155):1349-1357.

4. Timor Tritsch IE, Monteagudo A. Unforeseen consequences of the increasing rate of cesarean deliveries: early placenta accrete and cesarean section scar pregnancy; a review. Am J Obstet Gynecol. 2012;207(1):14 29.

5. Vial Y, Petignat P, Hohlfeld P. Pregnancy in a cesarean scar. Ultrasound Obstet Gynecol. 2000;16(6):592-593.

6. Timor Tritsch IE, Monteagudo A, Santos R, et al. The diagnosis, treatment, and follow-up of cesarean scar pregnancy. Am J Obstet Gynecol. 2012;207(1):44.e1-13.

7. Mosad A, Altraigey A. Scar pregnancy and spontaneous rupture uterus a case report. Ginekologia Polska. 2017;88(12):698-699.

8. Rotas MA, Haberman S, Levgur M. Cesarean scar ectopic pregnancies: etiology, diagnosis, and management. Obstet Gynecol. 2006;107(6):13731381.

9. Seow KM, Hwang Jl, Tsai YL, et al. Subsequent pregnancy outcome after conservative treatment of a previous cesarean scar pregnancy. Acta Obstet Gynecol Scand. 2004;83(12):1167-1172.

10. Shufaro Y, Nadjari M. Implantation of a gestational sac in a cesarean section scar. Fertil Steril. 2001; 75(6):1217.

11. Veridiano NP, Lopes $\mathrm{J}, \mathrm{Ohm} \mathrm{HK}$, et al. Placenta percreta as a cause of uterine perforation during abortion. A case report. J Reprod Med. 1986;31(11):1049-1050.

12. Herman H, Weinraub Z, Avrech 0, et al. Follow up and outcome of isthmic pregnancy located in a previous cesarean section scar. Br J Obstet Gynecol. I995:102(10);839-841.

13. Rempen A, Albert P. Diagnosis and therapy of early pregnancy implanted in the scar of cesarean section. Z Geburtshilfe Perinatol. 1990;194(1):4648.

14. Fylstra DL. Ectopic pregnancy within a cesarean scar: a review. Obstet Gynecol Surv. 2002;57(8):537-543.

15. Seow KM, Hwang JL, Tsai YL. Ultrasound diagnosis of a pregnancy in a cesarean section scar. Ultrasound Obstet Gynecol. 2001;18(5):547-549.

16. Lai YM, Lee JD, Lee CL, et al. An ectopic pregnancy embedded in the myometrium of a previous cesarean section scar. Acta Obstet Gynecol Scand. 1995;74(7):573-576.

17. Godin PA, Bassil S, Donnez J. An ectopic pregnancy developing in a previous caesarian section scar. Fertil Steril. 1997;67(2):398-400.

18. Maymon R, Halperin R, Mendlovic S, et al. Ectopic pregnancies in caesarean section scars: the 8-year experience of one medical centre. Hum Reprod. 2004;19(2):278-284.

19. Hung TH, Shau WY, Hsieh TT, et al. Prognostic factors for an unsatisfactory primary methotrexate treatment of cervical pregnancy: a quantitative review. Hum Reprod. 1998;13(9):2636-2642.

20. Ayoubi JM, Fanchin R, Meddoun M, et al. Conservative treatment of complicated cesarean scar pregnancy. Acta Obstet Gynecol Scand. 2001;80(5):469-470.

21. Ravhon A, Ben-Chetrit A, Rabinowitz R, et al. Successful methotrexate treatment of a viable pregnancy withina thin uterine scar. Br J Obstet Gynaecol.1997;104(5):628-629. 
22. Shufaro Y, Nadjari M. Implantation of a gestational sac in acesarean section scar. Fertil Steril. 2001; 75(6):1217.

23. Nawroth F, Foth D, Wilhelm L, et al. Conservative treatment of ectopic pregnancy in a cesarean section scar with methotrexate: a case report. Eur J Obstet Gynecol Reprod Biol. 2001;99(1):135-137.

24. Wu X, Zhang X, Zhu J, et al. Caesarean scar pregnancy: comparative efficacy and safety of treatment byuterine artery chemoembolization and systemic methotrexate injection. European Journal of Obstetrics \& Gynecology and Reproductive Biology. 2012;161(1):75-79.
25. Yang XY, Yu H, Li KM, et al. Uterine artery embolisation combined with local methotrexate for treatment of caesarean scar pregnancy. BJOG. 2010;117(8):990-996.

26. Chen FY, Torng P, Huang S, et al. Ectopic pregnancy withina cesarean section scar: report of two cases. Taiwanese $J$ Obstet Gynecol. 2004;43(1):38-41.

27. Vial Y, Petignat P, Hohlfeld P. Pregnancy in a cesarean scar. Ultrasound Obstet Gynecol. 2000;16(3):592-593. 